\title{
Erroneous message discovery with data acquisition and secret communication in cellular networks
}

\author{
R. Balakrishna ${ }^{1 *}$, R. Anandan ${ }^{2}$, A. Sajeev Ram ${ }^{3}$ \\ ${ }^{1}$ Department of Computer Science \& Engineering, Vels Institute of Science, Technology and Advanced Studies (VISTAS), Chennai, India. \\ ${ }^{2}$ Department of Computer Science \& Engineering, Vels Institute of Science, Technology and Advanced Studies (VISTAS), Chennai, India. \\ ${ }^{3}$ Department of Computer Science \& Engineering, Vels Institute of Science, Technology and Advanced Studies (VISTAS), Chennai, India. \\ *Corresponding authorE-mail:krishna.se@velsuniv.ac.in
}

\begin{abstract}
Cellular networks are susceptible to different varieties of security barrages, inclusive of erroneous message inoculation, message falsification and monitoring. Sensitivity knots can be adjudicate by invaders and the adjudicate knots can misinterpret message integrity by inoculating erroneous message. Erroneous message can be inoculated by adjudicate sensitivity knots in different measures, Inclusive of data acquisition and broadcasting. In a System the erroneous message exposure methods contemplate the erroneous message inoculations during the message promoting only. In an (SEP) symmetric en-route purifying schemes facilitates broadcasting knots and central terminal will expose erroneous message with a assertive possibility. In an interlaced step-by-step validation pattern Sensitivity knots are not granted to execute the data acquisition during message promoting. The Capricious Cipher based En-route purifying scheme (CCEP) nips erroneous message en-route without balanced key distribution. Message confidentiality means message to be enciphered at the origin knot and deciphered at the terminal. However, message acquisition methods usually need any enciphered sensitivity message to be deciphered at data acquisition. The essential idea at the rear of the erroneous message exposure algorithm is to make team of sensitivity knots in which one team operates a message authentication code (MAC) of promoting message and the alternate team afterwards checks the message using the MAC. Data acquisition is equipped in cellular sensitivity network in order to remove message repetition, minimize message communication, and increase message efficiency.
\end{abstract}

Keywords: Cellular networks, sensitivity knots, cipher, data acquisition, encipher, decipher, data exposure.

\section{Introduction}

The elected viewpoint of data acquisition and Verification protocol (DAV) is to give erroneous message exposure [1] and protect data acquisition against up to $\mathrm{N}$ adjudicate sensitivity knots, for $\mathrm{N}>1$ The value of $\mathrm{N}$ depends on earnest specifications, Knot quantity, Folder dimensions and the quantity of sustainable overload. We speculate that certain sensitivity knots were picked influentially as fact acquisition along with the knots among couple of continues fact generators are named promoting knots as they promote message. In order to expose erroneous message inoculated by fact acquisition during fact acquisition, as a result nearby knots of the fact acquisition(named observing knots) too operate fact acquisition and also operate MACs for the acquisition message to provide their team to check the message afterwards [2]. DAV equally gives message privacy as messages were promoted among fact acquisitions. In order to afford message privacy while message promoting among each couple of continuous data acquisitions, the acquainted message are enciphered at data acquisitions and erroneous message exposure is implemented over the enciphered message rather than the transparent message. Whenever the authentication of enciphered message drops at a promoting knots, the message are discarded promptly to reduce the fritter of properties like broadband and accumulator mechanization causes erroneous message inoculation [5]. The mainstay of this work is to integrate the erroneous message exposure with Data Acquisition and
Confidentiality Using data acquisition and verification (DAV) protocol.

In the Existing System the erroneous message exposure methods consider the erroneous message inoculation during the message promoting only. In an SEP techniques [7] provides broadcasting knots along with central terminal for expose erroneous message under a assertive possibility. In interlaced step-by-step verification techniques, Sensitivity knots were not supposed to conduct the fact acquisition while message promoting. The Capricious Cipher oriented En-route Purifying method (CCEP) nips erroneous message en-route without balanced key distribution [9]. The major disadvantages are as follows,

- In a big-scale sensitivity network respective sensitivity knots are exposed to security adjudicates.

- Networks are susceptible to event fiction attacks, in which the adjudicate knots inoculate fake details into the network.

- Attacks by adjudicated sensitivity knots could bring in not only erroneous dismay along with that reduction of the fixed quantity of power in a accumulator mechanized system.

In the current work Data Acquisition and Verification protocol (DAV), to integrate the erroneous message exposure with the data acquisition and confidentiality is performed to overtake those challenges in the existing system. To back the data acquisition along with erroneous message exposure, the observing knots of each fact acquisition too perform the fact acquisition along with perform the respective modest-amount data verification digit for message authentication in the teammates. To aid privacy message 
communication, the sensitivity knots among couple of continuous fact acquisition to check the message uniqueness on the enciphered message and not in the transparent message. The major advantages of this effort are as follows,

- Symmetric en-route purifying (SEP) schemes is used to expose the erroneous message and detail it.

- By using the teammates operation we can send and receive the data safely

- Data acquisition is performed in cellular sensitivity network and it removes message repetition, minimize message communication and increase message accuracy [4].

\section{Related work}

\section{A Survey on sensor networks}

Cellular sensitivity Networks are gently approved in the manufacturing sector because of their enhancement on connected systems [6]. Along with the preserving connecting rates, cellular networks enlarge the domain of backgrounds achievable to observe. Therefore by the sum of sensor along with assisting potentials to opposed in the corporal sectors and make for transmitting among these objects or along with resources in the near forthcoming web. However, the acmessagement of cellular networks by the manufacturing computerization group is disrupted by main problems, such as privacy providence and warranty of best services. To test the above two criteria, we pick to inspect necessary cellular network methodologies which is meant for the manufacturing computerization. We examine best of services requirements in order to carry out a menace analysis, which is important for our validation. According to the results of this validation, we found and examine open research issues.

\section{Symmetric en-route purifying inoculated erroneous message in sensitivity networks}

In a big-scale sensitivity network particular sensors are subject to security adjudicates. A adjudicated knot can inoculate fictitious sensing details into the network. If undetected, these fictitious details would be promoted to the message gathering point (i.e. the wreck). Such attacks by adjudicated sensing can motive erroneous dismay as well as reduction of the limited quantity of power in a accumulator mechanized system [7]. In this paper we shows a Symmetric oriented Purifying (SEP) operation which can expose along with removal of erroneous details. SEP needs that each sensitivity details are tested by important message authentication codes (MACs), each created by a knot which exposes the similar process. Now the detail is promoted, every knot through the path checks the effectiveness of the MACs possibilities which leaves any invalid MACs at primitive end occurs. The wreck continues to purify the available erroneous details which are not detected in the en-route purifying. SEP accomplishes the system range in order to find the uniqueness of every detail by means of cumulative rulesformulating in the manner of exposing knots along with cumulative erroneous details exposure by many promoting knots. Our analysis and reproductions show that, under an overload of 16 bytes per detail, SEP is capable of minimizing $85-95 \%$ erroneous inoculated details by a adjudicated knot within 10 promoting steps.

\section{Interleaved step-by-step verification against erroneous message inoculation attacks in sensitivity networks}

Sensitivity systems generally expand in neglected regions, therefore disappearing these systems susceptible for erroneous message inoculation assaults in an intruder inoculates erroneous message in an systems with the aim of betraying the central terminal or reducing the materials of the broadcasting knots [8] Unified verification methods will not protect this assault in case the attacker has adjudicated more than one or a less amount of sensing knots. We showcased three interlaced step-by-step verification methods which assures the central terminal can expose inoculated erroneous message quickly which is not greater than $\mathrm{N}$ knots were adjudicated, where $N$ is a system structure variable. Additionally, these methods will provide in between promoting knots to expose and remove erroneous message container as soon as possible. Our performance measures indicate that our technique is more feasible along with the protection it gives and it also permits adjustment among protection and throughputs. A blueprint execution of this technique shows, technique is hands-on and can be expand on the sensing knots.

\section{Materials and methods}

\section{Data acquisition and verification protocol (DAV)}

A cellular network with densely expand sensing knots, In that certain knots are designed as fact acquisition. Let us consider a amount for $\mathrm{N}$, fact acquisition actually picked in a manner that (i) $\mathrm{N}$ knots will occur among couple of fact acquisition and (ii) every fact acquisition will have $\mathrm{N}$ nearby knots. The result of this algorithm is though the network can have up to $\mathrm{N}$ arbitrated knots, message is assembled in fact acquisitions, fact privacy is given along with the inoculated erroneous message were exposed as well as removed [12]. The following steps carried out in this algorithm,

- $\quad \mathrm{N}$ nearby knots of each fact acquisition is randomly picked as observing knots in order to operate the additional fact acquisition as well as to operate subMACs in the gathered message.

- The subsequent $2 \mathrm{~N}+1$ team of knots were created in the way each the knot of all team to stake a definite symmetric key. (1) First team is created by the present as well as promoted fact generators. (2). $\mathrm{N}$ teams were created by the observing knots of the present fact acquisition, Finally (3). N teams are created by the observing and promoting knots of the present fact generator

- Each data acquisition and the picked $\mathrm{N}$ observing knots gather message and then operate subMACs. The gathered fact are enciphered by the present fact acquisition. The fact acquisition along with its observing knots operates couple subMACs. First SubMacs for the enciphered acquainted message and another subMAC for the transparent message.

\section{Observing knot selection algorithm}

The input for this algorithm are Aggregator AU, its n neighboring nodes, the group key $\mathrm{K}$ and the output are as follows,

$\mathrm{N}$ nearby knots of ' $\mathrm{a}$ ', are selected as observing knots.

- A request its nearby knots to send two arbitrary digits along with its knot ID digit.

- $\quad$ Each nearby knots of A creates two arbitrary digits (Pa and $\mathrm{Pb}$ ) utilizes PRING along with the key it stakes among A. $\mathrm{Pa}, \mathrm{Pb}$ along with $\mathrm{MAC}(\mathrm{Pa} \mid \mathrm{Pb})$ were transferred to A.

- When ' $a$ ' finishes getting arbitrary digits and knot IDs from $q$ its nearby knots, it marks them qi in the receiving order of their arbitrary digits first and foremost are marked as q1 and qn respectively.

- $\mathrm{Au}$ sorts all $2 * \mathrm{q}$ arbitrary digits in an ascending order. $\mathrm{P} 1, \mathrm{P} 2 \ldots \mathrm{P} 2 * \mathrm{n}$ and compute MAC $(\mathrm{P} 1 \mid \mathrm{P} 2) \ldots \mid \mathrm{P} 2 * \mathrm{n})$ using $\mathrm{k}$ group. Then $\mathrm{Au}$ broadcast the sorted arbitrary digits and MAC k group $(\mathrm{P} 1|\mathrm{P} 2| \ldots . . \mid \mathrm{P} 2 * n)$ along with knots IDs and their new marks.

- Each Qi checks the broadcast digits by verifying whether two arbitrary digits $\mathrm{Pa}$ and $\mathrm{Pb}$ that it sent earlier to $\mathrm{Au}$ match two of the arbitrary digits that $\mathrm{Au}$ has broadcasted [12]. 
The function of above two algorithms for securely transferring the data has been explained clearly in Fig 1 .

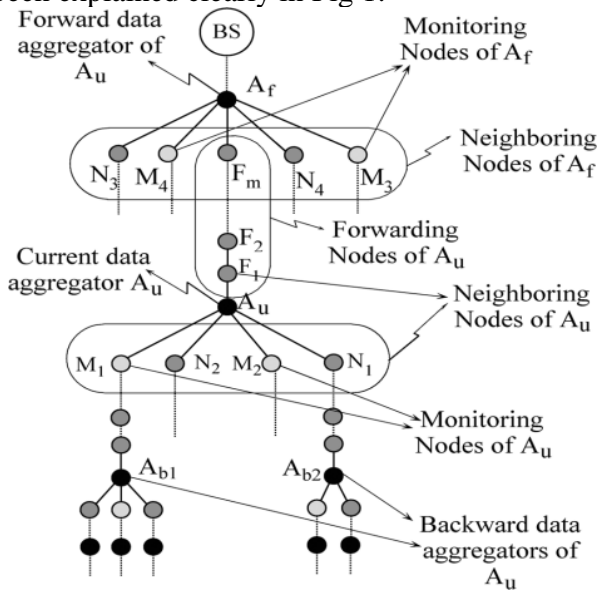

Fig. 1: Architecture diagram

\section{Network topology}

The data generators are selected in a manner that: 1) In the cellular systems there will be $\mathrm{N}$ knots, called promoting knots, through any couple of continuous fact generators along with 2) every fact generators will have $\mathrm{N}$ nearby knots, As a result $\mathrm{N}$ pairs are created with the promoting knots on the way between couple of continuous fact acquisitions .

\section{Observing knot selection}

Each data generator is observed by its $\mathrm{N}$ nearby knots out of total $\mathrm{n}$ nearby knots. $\mathrm{N}$ nearby of a data acquisition $\mathrm{A}$ are picked as observing knots to compute the fact acquisition in order to operate subMACs on the generated fact. Observing knots were picked through Observing Knot Selection (OKS) method. The selection of $\mathrm{N}$ observing knots for every fact generators in method OKS is to provide indices for nearby knots in some order and then operate $\mathrm{N}$ indices by performing modulus calculation to the total of among arbitrary digits created by the nearby knots. The fact generators along with nearby knots are there in the picking of observing knots to reduce the effects of a adjudicated knots.

\section{Team formation}

The subsequent $2 \mathrm{~N}+1$ team of knots were created in the way each the knot of all team to stake a definite symmetric key.

- First team is created by the present fact generator along with the promoting fact generator (AA-type).

- $\quad \mathrm{N}$ team is created by the observing along with promoting knots of the present fact generator (MF-type).

- $\quad \mathrm{N}$ teams were created by the observing knots of the present fact generator along with nearby knots of the promote fact generator (MN-type)

\section{Data acquisition and erroneous message exposing}

Each data generator and its picked $\mathrm{N}$ observing knots gather data and then operate subMACs. The aquatinted message is enciphered by the present fact generator. The fact generator along with its observing knots operates couple of subMACs: first subMAC for the enciphered message and another subMAC for the transparent message. The present fact generator creates couple of FMACs from these subMACs along with that it transfers the enciphered message and two FMACs to promoting knots. The integrity of the enciphered is checked by promoting teams of the picked observing knots of the present fact generator. The purity of the transparent message is checked by some nearby knots of the promoted data generation. If the purity checked for enciphered or transparent message drops at any sensor knots, the message is deleted instantly.

\section{Results and discussion}

\section{Selection of data aggregator and monitoring node selection}

In order to Transfer the message from source to destination securely we have to select Data Aggregator and monitoring nodes in the wireless senor networks.
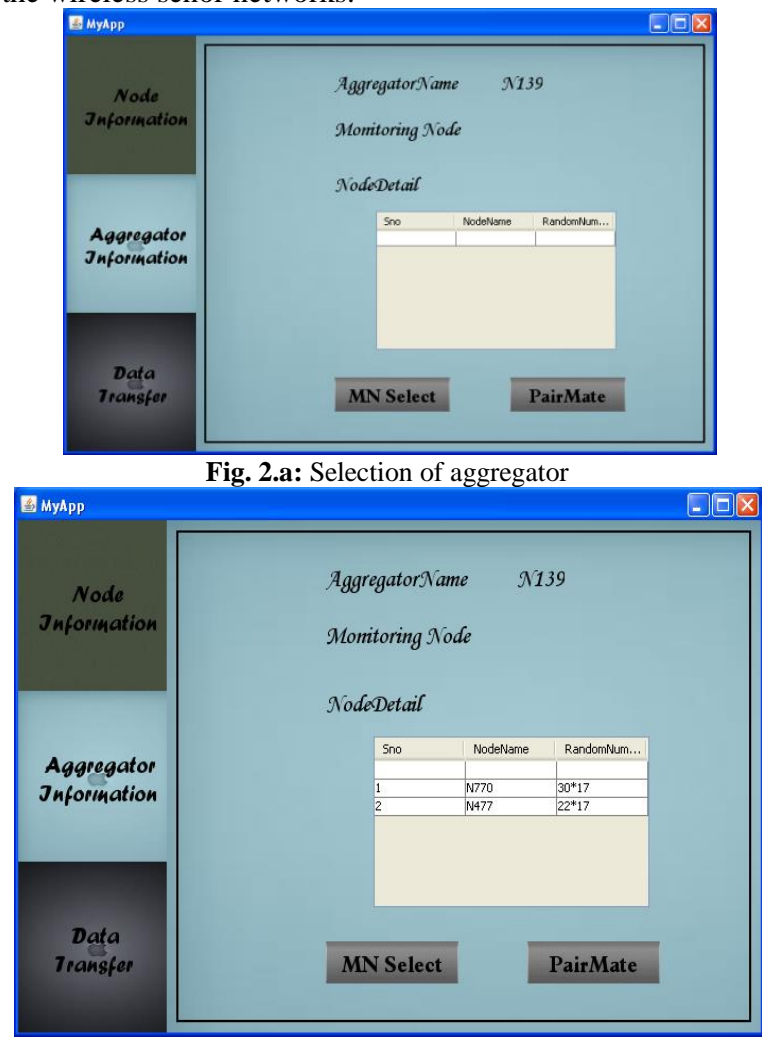

Fig. 2.b: Selection of monitoring nodes

\section{Transfer of message by paring the nodes}

Once the Data Aggregator and monitoring nodes are selected, our next step is to form a pair (AA, MF, MN type) between two nodes for transferring the messages using secret keys.

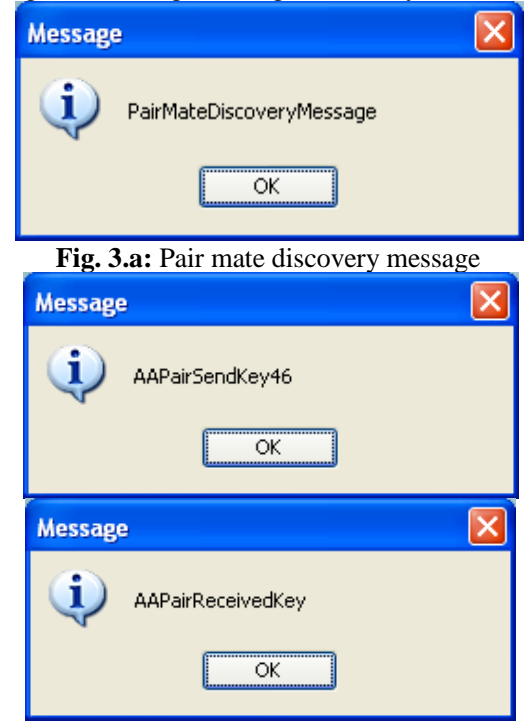

Fig. 3.b: Sharing of key between AA Pair 

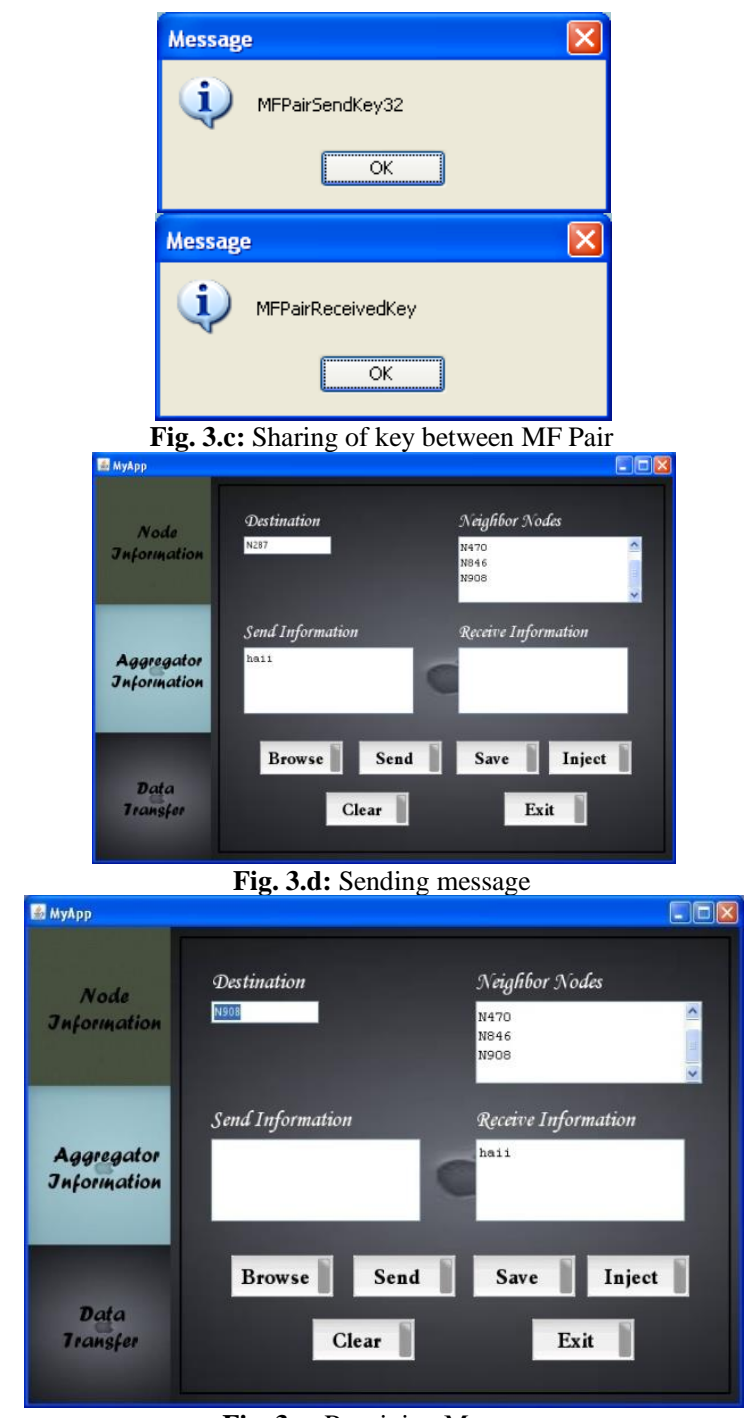

Fig. 3.e: Receiving Message

\section{Conclusion}

In cellular networks adjudicated sensing knots the purity of message, by inoculating erroneous message. However, this research has shown the unique protection protocol DAV to combine fact acquisition privacy and erroneous message revelation. DAV adjoins couple of FMACs to every message content. To minimize the communication aerial, the quantity of every FMAC is finite. Every FMAC contains subMACs to protect the message against up to adjudicated sensing knots. The yield measures showcase that the operational along with transfer of message aerial of DAV were not significant, As a result creating the employing of DAV reasonable. The simulation output indicates that the quantity of communicated messages is minimized in the range of $60 \%$, Which causes Compelling enhancement in bandwidth exertion and power utilizations.

\section{Future enhancement}

As for the future research, consider of sanctioning every sensing knot to be capable of both generating and promoting messages for upgrading system protection along with performance. Previously known methods on erroneous message exposure which do not support fact privacy along with acquisition, even though they are generally efficient to cellular systems.

\section{References}

[1] Nii E, Kitanouma T, Adachi N \& Takizawa Y, "Cooperative detection for falsification and isolation of malicious nodes for wireless sensor networks in open environment", IEEE Asia Pacific Microwave Conference (APMC), 2017, pp.521-524.

[2] Jose J, Jose J \& Muhammed Ilyas H, "Symmetric concealed data aggregation techniques in wireless sensor networks using Privacy Homomorphism: A review", International Conference on Information Science (ICIS), (2016), pp.275-280.

[3] Dobslaw F, Gidlund M \& Zhang T, "Challenges for the use of data aggregation in industrial Wireless Sensor Networks", IEEE International Conference on Automation Science and Engineering (CASE), (2015), pp.138-144.

[4] Bharuka K \& Jinwala DC, "A secure data aggregation protocol for outlier detection in wireless sensor networks using aggregate Message Authentication Code", 9th International Conference on Industrial and Information Systems (ICIIS), (2014), pp.1-6.

[5] Ozdemir S \& Cam H, "Integration of False Data Detection With Data Aggregation and Confidential Transmission in Wireless Sensor Networks", IEEE/ACM Transactions on Networking, Vol.18, No.3, (2010).

[6] Akyildiz F, Su W, Sankarasubramaniam Y \& Cayirci E, "A Survey on sensor networks", IEEE Commun. Mag., Vol.40, No.8, (2002), pp.102-114.

[7] Ye F, Luo H, Lu S \& Zhang L, "Statistical en-route detection and filtering of injected false data in sensor networks", Proc. IEEE INFOCOM, Vol.4, (2004), pp.2446-2457.

[8] Zhu S, Setia S, Jajodia S \& Ning P, "Interleaved hop-by-hop authentication against false data injection attacks in sensor networks", ACM Trans. Sensor Netw., Vol.3, No.3, (2007).

[9] Yang H \& Lu S, "Commutative cipher based en-route filtering in wireless sensor networks", Proc. IEEE VTC, (2004),pp.1223-1227.

[10] $\mathrm{Yu} \mathrm{Z} \&$ Guan $\mathrm{Y}$, "A dynamic en-route scheme for filtering false data in wireless sensor networks", Proc. IEEE INFOCOM, Barcelona, Spain, (2006), pp.1-12.

[11] Intanagonwiwat C, Estrin D, Govindan R \& Heidemann J, "Impact of network density on data aggregation in wireless sensor networks", Proc. 22nd Int. Conf. Distrib. Comput. Syst., (2002), pp.575-578.

[12] Perrig A, Szewczyk R, Tygar JD, Wen V \& Culler DE, "SPINS: Security protocols for sensor networks", Wireless networks, Vol.8, No.5, (2002), pp.521-534. 